\title{
A fatal combination of situs inversus, pregnancy and cardiac arrest treated with an automated external defibrillator
}

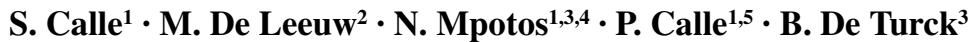

Published online: 23 May 2016

(C) The Author(s) 2016. This article is available at SpringerLink with Open Access

\begin{abstract}
Answer
The AED delivered wrongful shocks, probably because the tachyarrhythmia with QRS complexes of low amplitude was considered to be ventricular fibrillation. Evidence for this comes from the ECG strips from the first AED rhythm analysis, clearly showing QRS complexes of a similar shape and amplitude, but at a lower rate. Furthermore, these small QRS complexes were continuously found from the start of the resuscitation attempt until the fourth shock (data not shown).

This case illustrates that the specificity of shock/noshock decisions by the AEDs is not $100 \%$ [1, 2]. Cardiologists should also be aware that AED sensitivity figures are only within a 90-95\% range, and that external artefacts during the analysis process (such as chest compressions) decrease the accuracy significantly. This implies that shock/no-shock decisions should be scrutinised before being used for diagnostic/therapeutic decision making and prognostication.
\end{abstract}

S. Calle

simon.calle@ugent.be

1 Faculty of Medicine and Health Sciences, Ghent University, Ghent, Belgium

2 Forensic Pathology Department, Ghent University Hospital, Ghent, Belgium

3 Department of Emergency Medicine, Sint Lukas General Hospital, Ghent, Belgium

4 Faculty of Medicine and Health Sciences, University of Antwerp, Wilrijk, Belgium

5 Department of Emergency Medicine, Maria Middelares General Hospital, Ghent, Belgium
Remarkably, no cause of death could be detected on autopsy. Therefore, further research on the cardiac arrest risk stratification in dextrocardia in general, and during pregnancy in particular, is needed.

A third lesson concerns the low amplitude of QRS complexes detected by the AED electrodes placed in the conventional sternal-apical position [3]. Therefore, it seems reasonable in known dextrocardia cases to change to bi-axillary electrodes placement or a mirror-like approach (i.e. placement to the left of the sternum and in the right midaxillary line). The reduced QRS amplitude in this dextrocardia case also argues against the use of the abovementioned mirror-like approach in a standard cardiac arrest patient with a medical device implanted below the right clavicle.

Open Access This article is distributed under the terms of the Creative Commons Attribution 4.0 International License (http:// creativecommons.org/licenses/by/4.0/), which permits unrestricted use, distribution, and reproduction in any medium, provided you give appropriate credit to the original author(s) and the source, provide a link to the Creative Commons license, and indicate if changes were made.

\section{References}

1. Calle PA, Mpotos N, Calle SP, Monsieurs KG. Inaccurate treatment decisions of automated external defibrillators used by emergency medical services personnel: incidence, cause and impact on outcome. Resuscitation. 2015;88:68-74.

2. Calle PA, Paepe P De, Van Sassenbroeck D, Monsieurs KG. External artifacts by advanced life support providers misleading automated external defibrillators. Resuscitation. 2008;79:482-89.

3. Cattermole G, McKay N. A case of dextrocardiac ventricular fibrillation arrest. Emerg Med J. 2006;23:147-48. 